\title{
Inter-individual variability of EEG features during microsleep events
}

Martin Golz, Computer Science Faculty, University of Applied Sciences, Schmalkalden, Germany, golz@hs-sm.de Adolf Schenka, Computer Science Faculty, Univ. Applied Sciences, Schmalkalden, Germany, a.schenka@hs-sm.de Florian Haselbeck, Computer Science Faculty, Univ. Applied Sciences, Schmalkalden, Germany, f.haselbeck@ hs-sm.de ${ }^{1}$ Martin Pauli, Computer Science Faculty, Univ. Applied Sciences, Schmalkalden, Germany, m.pauli@stud.hs-sm.de

The recognition of changes in brain state is a challenging task. This is especially the case for short-term requirements, e.g. when microsleep events of car drivers should be detected from the EEG. Machine learning methods have been successfully used for this purpose. To the best of our knowledge, this is the first time that results of a leave-one-subject-out cross-validation (LOSO CV) for microsleep detection are presented. It involves training on the data of all subjects except one and validating on the data of the subject held out. This procedure is performed for each individual subject. It simulates the case that in the future data of subjects will be analyzed that were not used for machine learning training and therefore asks how general the results of machine learning can be interpreted.

8-channel EEG data (Fp1, Fp2, A1, C3, C4, A2, O1, O3; common average reference) from two studies in our driving simulation laboratory with 31 participants were analyzed. In total, 3,623 microsleep events were included. The length of segments $(4 \mathrm{sec})$ and the time offset of their beginning until the onset of clearly visible behavioral signs of microsleep (1 sec) were empirically determined. Feature vectors consisted of logarithmic power spectral densities estimated directly using the modified periodogram method and averaged within spectral bands of $1 \mathrm{~Hz}$ width, starting at $0.1 \mathrm{~Hz}$ and ending at $40.1 \mathrm{~Hz}$. These parameters were also empirically determined using the optimized learning vector quantisation as the machine learning method. Support-vector machines with Gaussian kernels were used for the final results. They generated high computational load during training and during hyper-parameter optimization, but outperformed three other alternatively used machine learning methods with regard to accuracy.

Repeated random subsampling across all 31 individuals yielded mean accuracies of $95.0 \pm 0.5 \%$. The exclusion of each of the 31 individuals, i.e. LOSO CV, resulted in very different accuracies. The results of 12 individuals were better than $95.0 \%$; the results of another 16 individuals were better than $86.6 \%$. But in three individuals, the EEG features during microsleep could not be explained by the pool of EEG features of all other individuals; for them, detection accuracies were only $82.0 \%, 78.7 \%$, and $70.8 \%$.

A subsequent inspection of the recorded EEG showed that in two individuals a bad signal quality due to poor electrode attachment could be the cause and in one individual a very unusual behavior. In all phases of extreme sleepiness he showed unusually high and long-lasting eyelid activity which interfered with the recorded EEG in all channels.

Nevertheless, the individually diverse manifestation of the short-term EEG is possibly a fact. This has already been reported from studies on features of $30 \mathrm{~s}$ long EEG segments during sleep. In studies with monozygotic and bizygotic twins, evidence was found of high individuality and high heritability of EEG features. One group of authors concluded that the EEG could possibly be the most inheritable trait of humans.

\footnotetext{
${ }^{1}$ Now employed at Pre-development Smart Mobility \& Machine Learning, Audi Electronics Venture GmbH, Gaimersheim, Germany
} 


\section{Deep Learning for Prediction of Diaphragm Activity from the Surface Electro- myogram}

Niclas Bockelmann, Institute for Electrical Engineering in Medicine, Universität zu Lübeck, Lübeck, Germany, niclas.bockelmann@student.uni-luebeck.de

Jan Graßhoff, Institute for Electrical Engineering in Medicine, Universität zu Lübeck, Lübeck, Germany, j.grasshoff@uni-luebeck.de

Lasse Hansen, Institute of Medical Informatics, Universität zu Lübeck, Lübeck, Germany, hansen@imi.uni-luebeck.de Giacomo Bellani, Università degli Studi Milano Bicocca, Dipartimento di Medicina e Chirurgia, Milano, Italy, giacomo.bellani1@unimib.it

Mattias Heinrich, Institute of Medical Informatics, Universität zu Lübeck, Lübeck, Germany, heinrich@imi.uniluebeck.de

Philipp Rostalski, Institute for Electrical Engineering in Medicine, Universität zu Lübeck, Lübeck, Germany, philipp.rostalski@uni-luebeck.de

The electrical activity of the diaphragm (EAdi) is a novel monitoring parameter for patients under assisted ventilation and can be used for assessing the patient's neural respiratory drive. It is recorded by an array of electrodes placed inside the esophagus at the level of the diaphragm. This, however, mandates the insertion and proper positioning of a nasogastric catheter. A suitable noninvasive alternative is the measurement of the electromyogram by means of skin surface electrodes (sEMG) located on the thorax. The respiratory sEMG signal, however, is subject to electrocardiographic interference and crosstalk from other muscle and also located at a different spatial position with respect to the diaphragm. In this work we propose to use a deep convolutional neural network architecture to predict the electrical activity of the diaphragm as measured by a nasogastric catheter from surface EMG measurements. We adapt the ResNet architecture for efficient feature extraction from the sEMG signals and train our network to directly regress the EAdi as a supervised learning task. Given the high noise level of the input signals we further investigate a heatmap based regression approach. The proposed methods are evaluated on a clinical dataset consisting of 77 recordings from mechanically ventilated patients. For the direct regression task, the network's predictions reach a Pearson correlation coefficient (PCC) of 0.818 with EAdi on the holdout test set. The heatmap regression increases the PCC to 0.836 while at the same time achieving a lower root-mean-square error, indicating a superior performance in terms of absolute prediction accuracy. From our results we conclude that sEMG measurements can be used to predict the internal activity of the diaphragm as measured invasively using a nasogastric catheter. This confirms the findings of Bellani et al. that sEMG might be a viable method for noninvasively assessing respiration of mechanically ventilated patients. 


\section{Personalised training for automated sleep stage classification based on cardio- res-piratory features}

Sebastian Zaunseder, FH Dortmund, Dortmund, Germany, sebastian.zaunseder@fh-dortmund.de Lucas Weber, TU Dresden, Dresden, Germany, lucas.weber@mailbox.tu-dresden.de Miriam Goldammer, TU Dresden, Dresden, Germany, miriam.goldammer@tu-dresden.de

Supervised machine learning techniques play an increasingly important role for biomedical applications related to classification and prediction. Said techniques typically make use of large data sets to train a model for a specific purpose. While the common training strategy of using large datasets assures generalisation and robust functioning for unseen data on average, a trained model might fail to provide the ideal decision for an individual patient.

This work investigates the potential benefit of personalising classifiers by using an adjusted data set for training. As exemplary application we focus on sleep stage classification from cardiorespiratory features using the sleep heart health study. Different from the conventional training approach, we construct for each single patient an ideal subset of other patients for training. We identify such subsets in a supervised way by a sequential floating selection algorithm, which is known from feature selection.

It turns out that the personalised training subsets improve the classification compared to classifiers that uses all other patients by approximately $30 \%$. Thereby it typically uses less than $5 \%$ of the available patients for the training subset. The presented contribution clearly depicts the potential of a personalised training approach for classification tasks. However, by choosing the personalised training subset in a supervised way this work only serves to demonstrate the potential benefit of a personalised training subset. Future works have to focus on means to identify the subset members in an unsupervised way. 


\section{Comparison of Different Activation Functions of Neural Networks for Auto- regressive Modelling}

Marvin Schubert, Technische Hochschule Mittelhessen (THM), FB Life Science Engineering (LSE), Institut für Biomedizinische Technik (IBMT), Wiesenstr. 14, 35390 Gießen, Germany, e-mail: marvin.schubert@1se.thm.de Thomas Schanze, Technische Hochschule Mittelhessen (THM), FB Life Science Engineering (LSE), Institut für Biomedizinische Technik (IBMT), Wiesenstr. 14, 35390 Gießen, Germany, e-mail: thomas.schanze@1se.thm.de

An autoregressive model (AR model) is a special case of the autoregressive moving average model. Due to their properties, AR models are still often used to analyse time series and to provide short-term forecasts. The AR coefficients are usually estimated by solving the Yule-Walker equations or by least squares methods. When using an artificial neural network approach for classical AR modelling, the activation functions of the artificial neurons are linear. Our approach uses Google's TensorFlow to implement linear and non-linear neural network AR models. In addition to the use of least squares optimization or various learning rules to obtain the coefficients aka neurons s synaptic weights, L1 regularization or pruning of the coefficients can be applied in order to establish a sparse modelling or representation of the data. Of course, a key role is played by the activation function of an artificial neuron, which commonly defines the transfer of neuron's superimposed weighted post-synaptic input signals to neuron's output signal. This offers a simple possibility to do linear or non-linear AR processing of data. In this work, we focus on the investigation of the influence of various common non-linear activation functions, e.g. sigmoid or hyperbolic tangent function. For this purpose, we simulated noisy linear or non-linear AR signals. In addition, we used biomedical signals to test our approach. The results obtained for non-linear neurons are compared with those obtained for linear neurons and for the Yule Walker approach. Furthermore, we compare the different coefficient estimates and courses of cost functions for different regularization impacts. First results show, that our neural network approach to extend AR modelling towards non-linear and sparse AR modelling offers new possibilities for the analysis and representation of signals. 


\section{Development of an optimization based framework for online probabilistic fusion with an application to IMU-based magnetometer-free human motion capturing}

Michael Lorenz, Department of Computer Science, Technische UniversitDet Kaiserslautern, Kaiserslautern, Germany, lorenz@cs.uni-kl.de

Bertram Taetz, Department of Computer Science, Technische UniversitDet Kaiserslautern, Kaiserslautern, Germany, taetz@cs.uni-kl.de

Manon Kok, Delft Center for Systems and Control (DCSC), Delft University of Technology, Delft, The Netherlands, M.Kok-1@tudelft.nl

Markus Miezal, Department of Computer Science, Technische UniversitDet Kaiserslautern, Kaiserslautern, Germany, miezal@cs.uni-kl.de

Gabriele Bleser, Department of Computer Science, Technische UniversitDet Kaiserslautern, Kaiserslautern, Germany, bleser@cs.uni-kl.de

In inertial human motion capturing (IHMC), typically one inertial measurement unit (IMU) is placed on each segment that should be captured. By fusing the measurements with a suitable biomechanical as well as motion model, it is possible to capture the joint angles drift-free. However, magnetometer- and drift-free capturing of the global heading direction (as well as reliable global position estimates) are an active field of research, with mobile applications ranging from medicine and health over sports to ergonomics and workplace analysis.

Typical solutions fuse the sensor information using extended Kalman Filter (EKF) to obtain this information about human motion. Previous works showed that optimization based approaches outperform EKF based solutions when it comes to omitting magnetometer information, however, at the expense of increased computational complexity.

In this work, we present a magnetometer-free optimization based framework for IHMC. The core consists of a nonlinear least squares solver in a moving horizon fashion. The framework inherently scales from a (recursive) EKF to a Full Information Estimator and can thus be adapted to the situation at hand. The underlying algorithm is online-capable and can easily incorporate additional knowledge and models. Such models could result from first principles (e.g. physical laws), from data-based models, like with Gaussian processes or (Bayesian) neural networks, or from a combination of both types of models, known as grey-box models.

Regarding IHMC, this flexibility can be used to improve the estimate of relative and global joint angle estimates in challenging situations, e.g. if no magnetometer information is present.

The presented approach was tested on simulated and measured data from a lower body configuration given optical reference data. 


\section{Audio waves and its loss of energy in puncture needles}

Iván Maldonado, INKA, Institute of Medical Technology, Otto-von-Guericke University, Magdeburg, Germany, ivan.maldonado@ovgu.de

Alfredo Illanes, INKA, Institute of Medical Technology, Otto-von-Guericke University, Magdeburg, Germany, alfredo.illanes@ovgu.de

Axel Boese, INKA, Institute of Medical Technology, Otto-von-Guericke University, Magdeburg, Germany, axel.boese@ovgu.de

Marco Kalmar, INKA, Institute of Medical Technology, Otto-von-Guericke University, Magdeburg, Germany, marco.kalmar@ovgu.de

Thomas Suehn, INKA, Institute of Medical Technology, Otto-von-Guericke University, Magdeburg, Germany, thomas.suehn@ovgu.de

Michael Friebe, INKA, Institute of Medical Technology, Otto-von-Guericke University, Magdeburg, Germany, michael.friebe@ovgu.de

The interaction and location of a puncture needle's tip is crucial information for clinicians during a medical procedure; it can make the difference during the extraction of sample tissue or administration of a medicament. In most applications where a puncture needle is involved and imaging tools cannot provide useful feedback, the experience and skills from the clinician are essential to manipulate the direction of the tip and achieve a satisfactory result. Recent publications demonstrated that an audio transducer located at the proximal end of a needle is able to acquire relevant information from the distal tip-tissue interaction that is transmitted over the needle structure. The acquired audio signal can detect the transition between two tissue layers with different physical characteristics. This simple approach can be implemented in real applications as an assistant but it is indispensable to understand the correlation between an event on the needle tip, e.g. passing a layer, the surrounding parameters, e.g. insertion depth, and audio record. This work evaluates this correlation on simplified experimental setups. In a first test, the differences in reaction forces on a defined deformation of muscle and fat tissue were investigated using a bending test. Then a needle connected with an audio sensor was placed in various depths in meat and an impact with the help of a meat pendulum was given to the tip. This induces transversal and longitudinal waves that were acquired with the audio sensor. The signal was affected by the tissue's surrounding forces based on insertion depth. Finally, a correlation of insertion depth and the loss of energy in the decay of the audio signal was demonstrated. The quantity of energy loss per time unity was directly proportional to the needle's depth inside the tissue. The future of audio transducer's implementation is aimed at the characterization of tissue. 